\title{
Exact solutions for the spatial de Vaucouleurs and Sérsic laws and related quantities
}

\author{
A. Mazure ${ }^{1}$ and H. V. Capelato ${ }^{2}$ \\ 1 LAM Traverse du Siphon, BP 8, 13376 Marseille Cedex 12, France \\ 2 INPE/DAS CP 515, S. J. Dos Campos SP 12201-970, Brazil \\ e-mail: hugo@das.inpe.br
}

Received 20 September 2001/ Accepted 23 November 2001

\begin{abstract}
Using the Mathematica package, we find exact analytical expressions for the so-called de-projected de Vaucouleurs and Sérsic laws as well as for related spatial (3D) quantities - such the mass, gravitational potential, the total energy and the central velocity dispersion - generally involved in astronomical calculations expressed in terms of the Meijer G functions.
\end{abstract}

Key words. galaxies: elliptical and lenticular, $\mathrm{cD}$ - galaxies: fundamental parameters - galaxies: clusters: general

\section{Introduction}

Dynamical studies of astronomical systems like Elliptical Galaxies or Clusters of Galaxies involve the de-projection of observed (projected on the sky) quantities like surface brightness profiles, numerical density profiles, velocity dispersion profiles etc. The 3D profiles obtained are then used to derive e.g. the total luminosity (or mass) of the system or the gravitational potential and are used in the Jeans equation which is then resolved to get for instance the kinematics of the system.

The de Vaucouleurs profile (de Vaucouleurs 1948) and its generalization by the Sérsic law (Sérsic 1968), is one of the most often used laws particularly in the study of Elliptical Galaxies.

Unhappily these laws have so far lead to non-analytical de-projected (i.e. spatial) quantities. Efforts have been made in the last decades to provide either numerical tables (Poveda et al. 1960; Young 1976) or approximations and asymptotic expressions (Mellier \& Mathez 1987; Ciotti 1991; Graham \& Colless 1997; Ciotti \& Bertin 1999; Marquez et al. 2001).

Here we give analytical exact expressions for 3D quantities usually derived when using the de Vaucouleurs or Sérsic laws.

\section{Principles}

The classical de Vaucouleurs and Sérsic laws express the dependence on the projected central distance $R$ of for

Send offprint requests to: A. Mazure, e-mail: alain.mazure@astrsp-mrs.fr instance the Luminosity Intensity $I(R)$ of an Elliptical Galaxy.

The de Vaucouleurs law relates the intensity $I(R)$ to the central one, $I(0)$, by:

$I(R) / I_{0}=\exp \left[-7.66925\left[\left(R / R_{\mathrm{e}}\right)^{\frac{1}{4}}-1\right]\right]$

and the Sérsic profile, which is a generalization of the de Vaucouleurs profile, is written:

$I(R) / I_{0}=\exp \left[-b(m)\left[\left(R / R_{\mathrm{e}}\right)^{\frac{1}{m}}-1\right]\right]$.

The parameter $b(m)$ is determined from the definition of the effective radius $R_{\mathrm{e}}$, which is the projected radius inside which the projected luminosity (or mass) equals half of the total luminosity (or mass). The de Vaucouleurs law is recovered for $b(4)=7.66925$.

The requested $3 \mathrm{D}$ profiles are related to the derivative of the projected profiles by the usual Abel Integral written here for the $3 \mathrm{D}$ density profile $n(r)$ :

$n(r)=-\frac{1}{\pi} \int_{r}^{\infty} \frac{\mathrm{d} I}{\mathrm{~d} R} \frac{1}{\sqrt{R^{2}-r^{2}}} \mathrm{~d} R$.

Except for some particular cases there is no known exact expression for theses integrals, in particular in the case of the Sérsic (de Vaucouleurs) profiles.

However, using Mathematica we succeeded in obtaining exact analytical expressions for such integrals that involve the Meijer G functions. Physical quantities such as the spatial luminosity or mass profiles, the gravitational force, the gravitational potential and energy, which are combinations or integrals of the above functions, have also 
analytical expressions involving Meijer G functions. Some of these expressions are given below and numerical evaluations are compared to previous numerical calculations.

We first give the classical definition of the Meijer $G$ functions together with some of their properties which will be useful in understanding the results given by Mathematica.

\section{The Meijer functions}

These functions are defined as integrals of products of $\Gamma$ functions. The generalized Meijer $G$ function is defined as:

$G_{p, q}^{k, l}\left(z,\left.r\right|_{\beta_{1} \cdots \beta_{k}, \beta_{k+1} \cdots \beta_{q}} ^{\alpha_{1} \cdots \alpha_{l}, \alpha_{l+1} \cdots \alpha_{p}}\right)=\frac{r}{2 \pi i}$

$\int \frac{\left[\Gamma\left(1-\alpha_{1}-r u\right) \ldots \Gamma\left(1-\alpha_{l}-r u\right) \Gamma\left(\beta_{1}+r u\right) \ldots \Gamma\left(\beta_{k}+r u\right)\right]}{\left[\Gamma\left(\alpha_{l+1}+r u\right) \ldots \Gamma\left(\alpha_{p}+r u\right) \Gamma\left(1-\beta_{k+1}-r u\right) \ldots \Gamma\left(1-\beta_{q}-r u\right)\right]} z^{-u} \mathrm{~d} u$

(Gradshteyn \& Ryzhik 1980; Wolfram 1991; for a collection of formulae related to the Meijer $G$ functions cf. http://functions.wolfram.com/Hypergeometric Functions/MeijerG/).

The case $r=1$ defines the standard Meijer function, $\left.G_{p, q}^{k, l}\left(\left.z\right|_{\left\{\beta_{1} \cdots \beta_{k}\right\},\left\{\beta_{k+1} \cdots \beta_{q}\right\}} ^{\left\{\alpha_{1} \cdots \alpha_{l}\right\},\left\{\alpha_{l+1} \cdots \alpha_{p}\right\}}\right\}\right)$, which is the form we will be dealing with in the rest of this work. In the Mathematica StandardForm notation it writes as:

$$
\begin{aligned}
\text { Meijer } G\left[\left\{\left\{\alpha_{1} \cdots\right.\right.\right. & \left.\left.\alpha_{l}\right\}\left\{\alpha_{l+1} \cdots \alpha_{p}\right\}\right\} \\
& \left.\times\left\{\left\{\beta_{1} \cdots \beta_{k}\right\}\left\{\beta_{k+1} \cdots \beta_{q}\right\}\right\}, z\right] \\
& \equiv G_{p, q}^{k, l}\left(z \mid \begin{array}{l}
\left\{\alpha_{1} \cdots \alpha_{l}\right\},\left\{\alpha_{l+1} \cdots \alpha_{p}\right\} \\
\left\{\beta_{1} \cdots \beta_{k}\right\},\left\{\beta_{k+1} \cdots \beta_{q}\right\}
\end{array}\right) .
\end{aligned}
$$

For clarity we will keep both these notations, although suppressing the suffix "Meijer". Notice that in these formulae, the empty case: \{\} means that the corresponding coefficients are not defined and thus do not exist. This may occur, for instance, when $k=q$, or $l=p$, or else when one of those indices are null. An example is given by Eq. (7) below.

The following identity may be easily obtained by a substitution of the integration variable $u \rightarrow u+c$, where $c$ is a constant, in Eq. (4):

$$
\begin{aligned}
& G_{p, q}^{k, l}\left(z \mid \begin{array}{l}
\left\{\alpha_{1} \cdots \alpha_{l}\right\},\left\{\alpha_{l+1} \cdots \alpha_{p}\right\} \\
\left\{\beta_{1} \cdots \beta_{k}\right\},\left\{\beta_{k+1} \cdots \beta_{q}\right\}
\end{array}\right) \\
& \equiv z^{-c} G_{p, q}^{k, l}\left(z \mid \begin{array}{l}
\left\{\alpha_{1}+c \cdots \alpha_{l}+c\right\},\left\{\alpha_{l+1}+c \cdots \alpha_{p}+c\right\} \\
\left\{\beta_{1}+c \cdots \beta_{k}+c\right\},\left\{\beta_{k+1}+c \cdots \beta_{q}+c\right\}
\end{array}\right) .
\end{aligned}
$$

The moments of the Meijer function are expressible in terms of the higher-order Meijer functions:

$$
\begin{aligned}
& \int z^{\xi} G_{p, q}^{k, l}\left(\left.z\right|_{\left\{\beta_{1} \cdots \beta_{k}\right\},\left\{\beta_{k+1} \cdots \beta_{q}\right\}} ^{\left\{\alpha_{1} \cdots \alpha_{l}\right\},\left\{\alpha_{l+1} \cdots \alpha_{p}\right\}}\right) \mathrm{d} z= \\
& z^{-c} z^{1+\xi} G_{p+1, q+1}^{k, l+1}\left(\left.z\right|_{\left\{\beta_{1} \cdots \beta_{k}\right\},\left\{-(1+\xi), \beta_{k+1} \cdots \beta_{q}\right\}} ^{\left\{\alpha_{1} \cdots \alpha_{l}, \quad-\xi\right.}\right\},\{
\end{aligned}
$$

This may be straightforwardly demonstrated by inverting the order of the integrations.
In some particular cases the Meijer $G$ functions may be expressed in term of more classical special functions. As an example we give below the case of the Meijer function $G_{0,2}^{2,0}\left(z \mid \beta_{1}, \beta_{2}\right)$ (cf. http://functions.wolfram.com/07.09.03.0330):

$$
\begin{aligned}
G_{0,2}^{2,0}\left(z \mid \beta_{1}, \beta_{2}\right) & \equiv G_{0,2}^{2,0}\left(\left.z\right|_{\left\{\beta_{1}, \beta_{2}\right\},\{\}} \begin{array}{c}
\{\},\{\} \\
\end{array}\right) \\
& =2 z^{\left(\beta_{1}+\beta_{2}\right) / 2} K_{\beta_{1}-\beta_{2}}(2 \sqrt{z})
\end{aligned}
$$

where $K_{\tau}(x)$ is the modified Bessel function of $\tau$ - order.

\section{The 3D laws}

In the following, we give the analytical expressions using the Mathematica formalism for the $G$ Functions.

Let first start by introducing some useful dimensionless quantities. The dimensionless $2 \mathrm{D}$ and $3 \mathrm{D} x$ and $s$ radial distances are expressed in terms of $R_{\mathrm{e}}$ as:

$x \equiv R / R_{\mathrm{e}} ; \quad s \equiv r / R_{\mathrm{e}}$

The dimensionless $2 \mathrm{D}$ and $3 \mathrm{D}$ profiles are also defined as:

$i(x) \equiv I(R) / I_{0} \quad$ and $\quad \nu(s) \equiv n(r) \frac{R_{\mathrm{e}}}{I_{0}}$

which lets Eqs. (1) and (2) transform in the following reduced form:

$i(x)=\exp \left[-b(m)\left(x^{\frac{1}{m}}-1\right)\right]$

where the de Vaucouleurs law is obtained for $m=4$.

The de-projection integral (3) also transforms to:

$\nu(s)=-\frac{1}{\pi} \int_{s}^{\infty} \frac{\mathrm{d} i}{\mathrm{~d} x} \frac{1}{\sqrt{x^{2}-s^{2}}} \mathrm{~d} x$.

\subsection{De-projection of the de Vaucouleurs law}

We give here some detailed results for the case of the de Vaucouleurs law as an example and give more general results in the next section using the Sérsic law. Integrating the preceding equations using Mathematica, we first derive the expression for the 3D profile $n(r)$. We will then calculate the luminosity (or mass) profiles as well as the gravitational potential and the gravitational energy.

Only numerical estimations or asymptotic behaviors were given before (Poveda et al. 1960; Young 1976; Mellier \& Mathez 1987) so we will compare our results with those provided by Young for the spatial density and for the luminosity (or mass).

The basic ingredient to obtain the $\nu(s)$ profile is the derivative of the de Vaucouleurs law, Eq. (1), relative to the projected dimensionless distance, $x$, which is written:

$\frac{\mathrm{d} i}{\mathrm{~d} x}=-\frac{b}{4 x^{\frac{3}{4}}} \exp \left[-b\left(x^{\frac{1}{4}}-1\right)\right]$ with $b=7.66925$.

Integrating Eq. (11), the spatial density $\nu(s)$ expressed in terms of the dimensionless $3 \mathrm{D}$ radial distance $s$ is then 
Table 1. The spatial density and mass profiles for the de Vaucouleurs profile.

\begin{tabular}{lcccc}
\hline$s$ & $\nu(s)$ & $\nu_{\text {Young }}(s)$ & $M(s)$ & $M_{\text {Young }}(s)$ \\
\hline $1 \times 10^{-6}$ & $2.5553 \times 10^{6}$ & $2.5535 \times 10^{6}$ & $1.4730 \times 10^{-11}$ & $1.4717 \times 10^{-11}$ \\
$1 \times 10^{-5}$ & $3.5797 \times 10^{5}$ & $3.5786 \times 10^{5}$ & $2.1130 \times 10^{-9}$ & $2.1122 \times 10^{-9}$ \\
$1 \times 10^{-4}$ & $4.2189 \times 10^{4}$ & $4.2183 \times 10^{4}$ & $2.5959 \times 10^{-7}$ & $2.5954 \times 10^{-7}$ \\
$1 \times 10^{-3}$ & $3.7044 \times 10^{3}$ & $3.7042 \times 10^{3}$ & $2.4545 \times 10^{-5}$ & $2.4544 \times 10^{-5}$ \\
$1 \times 10^{-2}$ & $1.9679 \times 10^{2}$ & $1.9679 \times 10^{2}$ & $1.4961 \times 10^{-3}$ & $1.4960 \times 10^{-3}$ \\
$1 \times 10^{-1}$ & 4.4047 & 4.4047 & $4.4102 \times 10^{-2}$ & $4.4102 \times 10^{-2}$ \\
1. & $2.1943 \times 10^{-2}$ & $2.1943 \times 10^{-2}$ & $4.1536 \times 10^{-1}$ & $4.1536 \times 10^{-1}$ \\
10. & $7.8166 \times 10^{-6}$ & $7.8165 \times 10^{-6}$ & $9.4308 \times 10^{-1}$ & $9.4308 \times 10^{-1}$ \\
\hline
\end{tabular}

given by:

$$
\begin{aligned}
& \nu(s)=6.23828 s^{-7 / 4} G(\{\{\},\{\}\}, \\
& \left.\left\{\left\{\frac{1}{2}, \frac{5}{8}, \frac{3}{4}, \frac{7}{8}, \frac{7}{8}, 1, \frac{9}{8}, \frac{5}{4}\right\},\{\}\right\}, 0.713351 s^{2}\right) .
\end{aligned}
$$

One then obtains the luminosity (or mass) spatial profile defined by:

$M(s)=4 \pi \int_{0}^{s} s^{\prime 2} \nu\left(s^{\prime}\right) \mathrm{d} s^{\prime}$

which with a new formal integration gives:

$$
\begin{aligned}
& M(s)=39.1962 s^{\frac{5}{4}} G\left(\left\{\left\{\frac{3}{8}\right\},\{\}\right\},\right. \\
& \left.\left\{\left\{\frac{1}{2}, \frac{5}{8}, \frac{3}{4}, \frac{7}{8}, \frac{7}{8}, 1, \frac{9}{8}, \frac{5}{4}\right\},\left\{-\left(\frac{5}{8}\right)\right\}\right\}, 0.713351 s^{2}\right) .
\end{aligned}
$$

The gravitational potential is defined by:

$\Psi(s)=\int_{0}^{s} \frac{M\left(s^{\prime}\right)}{s^{2}} \mathrm{~d} s^{\prime}$

which gives:

$$
\begin{aligned}
& \Psi(s)=19.5981 s^{\frac{1}{4}} * \\
& G\left(\left\{\left\{\frac{3}{8}, \frac{7}{8}\right\},\{\}\right\},\left\{\left\{\frac{1}{2}, \frac{5}{8}, \frac{3}{4}, \frac{7}{8}, \frac{7}{8}, 1, \frac{9}{8}, \frac{5}{4}\right\},\right.\right. \\
& \left.\left.\left\{-\left(\frac{5}{8}\right),-\left(\frac{1}{8}\right)\right\}\right\}, 0.713351 s^{2}\right) .
\end{aligned}
$$

The values tabulated by Young (1976) are recovered defining the new potential:

$\Psi^{\prime}(s)=1-\Psi(s) / \Psi(\infty)$

such that

$\Psi^{\prime}(0)=1$ and $\left.\Psi^{\prime}(\infty)\right)=0$

In Table 1 , we compare the values given with the above expressions (normalized by the factor $\exp (b)\left(\pi 8 ! / b^{8}\right)$ ) to the numerical values obtained by Young for $s$ ranging from $10^{-6}$ to 10 .

\subsection{The de-projection of the Sérsic law}

We give now more general expressions for the 3D profile derived from the Sérsic law. This law is parametrized by an index $m$ and a parameter $b(m)$. The Sérsic law for $m=1$ corresponds to a $3-\mathrm{D}$ Exponential profile, already discussed by Fuchs \& Materne (1982) and for $m=4$ to the usual de Vaucouleurs law, treated in Sect. 2 above. In general, $b(m)$ is found as a solution of Eq. (A.4) given in the Appendix. Values of $b(m)$, (as well as $\log L(m)$ ) have also been given before by Ciotti et al. and Ciotti \& Bertin. We give in the appendix, values of $b(m)$ calculated using Mathematica for the range $m=1$ to $m=15$ and compared to those of Ciotti \& Bertin.

The spatial density expressed in terms of the dimensionless 3D distance $s$ can be written as (see e.g. Ciotti 1991; Graham \& Colless 1997):

$\nu(s)=\frac{b e^{b}}{\pi} s^{(1 / m-1)} \int_{0}^{1 .} \exp \left(-\frac{b s^{\frac{1}{m}}}{t}\right) \frac{1}{t^{2} \sqrt{t^{-2 m-1}}} \mathrm{~d} t(18)$

where $b$ means $b(m)$

Again this integral is found in terms of Meijer $G$ Functions which reduces to a Bessel function $\left(K_{0}\right)$ for $m=1$. We give below the various expressions for various values of $m$ and $b(m)$. Defining auxiliary constants:

$c_{1}(m) \equiv \frac{b(m) \exp [b(m)]}{(2 \pi)^{m} \sqrt{m}}$

and

$c_{2}(m) \equiv\left(\frac{b(m)}{2 m}\right)^{2 m}$

we obtain, for $m=1$ :

$\nu_{1}(s)=2 c_{1}(1) K_{0}(b(1) s)$.

For $m=2$ :

$2.58697 s^{-\frac{1}{2}}$

$\times G\left(\{\{\},\{\}\},\left\{\left\{0, \frac{1}{4}, \frac{1}{4}, \frac{1}{2}\right\},\{\}\right\}, 0.710231 s^{2}\right)$ 
For $m=4$ :

$5.26885 s^{-\frac{3}{4}} G(\{\{\},\{\}\}$,

$\left.\left\{\left\{0, \frac{1}{8}, \frac{1}{4}, \frac{3}{8}, \frac{3}{8}, \frac{1}{2}, \frac{5}{8}, \frac{3}{4}\right\},\{\}\right\}, 0.713351 s^{2}\right)$

Notice that Eq. (23) is equivalent to Eq. (13) for the de Vaucouleurs profile given before. This may be seen by applying the identity Eq. (5) and noting that $\beta_{\mathrm{S}}=$ $\beta_{\mathrm{DV}}-1 / 2$, where $\beta_{\mathrm{S}}$ and $\beta_{\mathrm{DV}}$ denote the arrays of $\beta$ coefficients appearing respectively in Eqs. (23) and (13).

The expressions above have the general form:

$$
\begin{aligned}
& \nu_{m}(s)=c_{1}(m) s^{\frac{1-m}{m}} G\left(\{\{\},\{\}\},\left\{\left\{\beta_{S}(m)\right\},\{\}\right\}, c_{2}(m) s^{2}\right)
\end{aligned}
$$

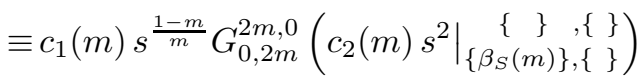

with $\beta_{S}(m)$ denoting the $2 m$-array:

$\beta_{S}(m) \equiv\left\{\left(\frac{j-1}{2 m}\right)_{1 \leq j \leq m} ;\left(\frac{j-2}{2 m}\right)_{m+1 \leq j \leq 2 m}\right\}$.

\subsection{Other related quantities}

Related quantities as the mass, the gravitational potential, the (total) potential energy and the (central) velocity dispersion, can then be formally calculated by other integrations, similar to what has been done for the de Vaucouleurs profile. From Eq. (14) we find for the mass:

$$
\begin{aligned}
& M(s)=2 \pi c_{1}(m) s^{\frac{2 m+1}{m}} G\left(\left\{\left\{-\frac{1}{2 m}\right\},\{\}\right\},\right. \\
& \left.\left\{\left\{\beta_{S}(m)\right\},\left\{-\frac{2 m+1}{2 m}\right\}\right\}, c_{2}(m) s^{2}\right) \\
& \equiv 2 \pi c_{1}(m) s^{\frac{2 m+1}{m}} G_{1,2 m+1}^{2 m, 1}\left(\left.c_{2}(m) s^{2}\right|_{\left\{\beta_{\mathrm{S}}(m)\right\},\left\{-\left(\frac{2 m+1}{2 m}\right)\right\}} ^{\left\{-\left(\frac{1}{2 m}\right)\right\}, \quad\{\}} .\right.
\end{aligned}
$$

Notice that in the case $m=1$, because of Eq. (21), an alternative expression for the mass may be given by:

$$
\begin{aligned}
M(s)= & 8 \pi c_{1}(1) \int_{0}^{s} z^{2} K_{0}(b \cdot z) \mathrm{d} z \\
= & \frac{8 \pi c_{1}(1)}{18} s^{3}\left[{ }_{2} F_{3}\left(\left\{\frac{3}{2}, \frac{3}{2}\right\},\left\{1, \frac{5}{2}, \frac{5}{2}\right\}, \frac{b^{2} s^{2}}{4}\right)\right. \\
& -\frac{3}{2}{ }_{1} F_{2}\left(\left\{\frac{3}{2}\right\},\left\{1, \frac{5}{2}\right\}, \frac{b^{2} s^{2}}{4}\right) \cdot \log \left(\frac{b^{2} s^{2}}{4}\right) \\
& \left.+\frac{9}{2} \sum_{j=0}^{\infty} \frac{2^{1-2 j} b^{2 j} s^{2 j} \Psi(0,1+j)}{(3+2 j) \Gamma^{2}(1+j)}\right]
\end{aligned}
$$

in which $b$ stands for $b(1)$ and $\Gamma(x)$ and $\Psi(n, x)$ denote the gamma function and its $(n+1)$ th derivative (i.e., the digamma - or $p s i$ - function in the case $n=0$, and polygamma function in the general case). Both expressions, Eqs. (26) and (27) have similar performances in Mathematica. The total mass, $M(\infty)=\frac{2 \pi}{b} \exp b$, is obtained to within $10^{-4}$, for $s=10$.

For the gravitational potential, using Eqs. (16) and (26), we find the following expression:

$$
\begin{aligned}
& \Psi(s)=\pi c_{1}(m) s^{\frac{m+1}{m}} * G\left(\left\{\left\{-\frac{1}{2 m}, \frac{m-1}{2 m}\right\},\{\}\right\},\right. \\
& \left.\left\{\left\{\beta_{\mathrm{S}}(m)\right\},\left\{-\frac{2 m+1}{2 m},-\frac{m+1}{2 m}\right\}\right\}, c_{2}(m) s^{2}\right) \\
& \equiv \pi c_{1}(m) s^{\frac{m+1}{m}} \\
& \times G_{2,2 m+2}^{2 m, 2}\left(\left.c_{2}(m) s^{2}\right|_{\left\{\beta_{S}(m)\right\},\left\{-\left(\frac{2 m+1}{2 m}\right),-\left(\frac{m+1}{2 m}\right)\right\}} ^{\left\{-\left(\frac{1}{2 m}\right),\left(\frac{m-1}{2 m}\right)\right\},\{\quad\}}\right) .
\end{aligned}
$$

Notice that, as before with the equations for $\nu(s)$ and for the same reasons, in the case $m=4$, Eqs. (26) and (28) above will differ from those given before in Sect. 4.1 (Eqs. (15) and (17)).

The gravitational potential energy is defined by:

$$
\Omega(s)=\frac{1}{2} \int_{0}^{s} \Psi(s) \mathrm{d} M(s)=\frac{1}{2} \int_{0}^{s} s^{2} \nu(s) \Psi(s) \mathrm{d} s .
$$

Unfortunately there seems to be no formal solution for this integral in terms of Meijer functions. However, by making use of a classical integral of a product of Meijer functions given in http://functions.wolfram.com/ 07.09.16.0025 one finds the following expression for the total potential energy:

$$
\begin{aligned}
& \Omega(\infty)=\frac{\pi c_{1}^{2}(m)}{4 c_{2}^{\frac{2+3 m}{2 m}}(m)} \cdot G\left(\left\{\left\{-\frac{1}{2 m}, \frac{1}{2}-\frac{1}{2 m}, \alpha_{E}(m)\right\},\{\}\right\}\right. \\
& \left.\quad\left\{\left\{\beta_{\mathrm{S}}(m)\right\},\left\{-1-\frac{1}{2 m},-1 / 2-\frac{1}{2 m}\right\}\right\}, 1\right) \\
& \equiv \frac{\pi c_{1}^{2}(m)}{4 c_{2}^{\frac{2+3 m}{2 m}}(m)} \\
& \times G_{2 m, 2 m+2,2 m+2}^{2 m}\left(\left.1\right|_{\left\{\beta_{\mathrm{S}}(m)\right\},\left\{-1-\frac{1}{2 m},-1 / 2-\frac{1}{2 m}\right\}} ^{\left\{-\frac{1}{2 m}, \frac{1}{2}-\frac{1}{2 m}, \alpha_{E}(m)\right\},\{\}}\right)
\end{aligned}
$$

where, besides the $2 m$-array of coefficients $\beta_{\mathrm{S}}(m)$ defined by Eq. (25), we have also defined the $2 m$-array: $\alpha_{\mathrm{E}}(m) \equiv$ $-(m+1) / m-\beta_{\mathrm{S}}(m)$.

The velocity dispersion of a spherical system in hydrostatic equilibrium is given by:

$\sigma^{2}(s)=\frac{1}{\nu(s)} \int_{s}^{\infty} \frac{M(x) \nu(x)}{x^{2}} \mathrm{~d} x$.

As for the gravitational potential energy, for systems endowed with a Sérsic density profile, this integral may be expressible in terms of Meijer functions for the case $s=0$. 
We proceed similarly as to Eq. (30) to get:

$$
\begin{aligned}
\sigma^{2}(0)= & \frac{1}{\nu(0)} \frac{\pi c_{1}^{2}(m)}{c_{2}^{1 / 2 m}(m)} \cdot G\left(\left\{\left\{-\frac{1}{2 m}, \alpha_{\sigma}(m)\right\},\{\}\right\},\right. \\
& \left.\left\{\left\{\beta_{\mathrm{S}}(m)\right\},\left\{-1-\frac{1}{2 m}\right\}\right\}, 1\right) \\
\equiv & \frac{1}{\nu(0)} \frac{\pi c_{1}^{2}(m)}{c_{2}^{1 / 2 m}(m)} \cdot G_{2 m+1,2 m+1}^{2 m, 2 m+1}\left(\left.1\right|_{\left\{\beta_{\mathrm{S}}(m)\right\},\left\{-1-\frac{1}{2 m}\right\}} ^{\left\{-\frac{1}{2 m}, \alpha_{\sigma}(m)\right\},\{\}}\right)
\end{aligned}
$$

with $\alpha_{\sigma}(m) \equiv(2 m-1) / 2 m-\beta_{\mathrm{S}}(m)$.

\section{Conclusions}

1. We obtain analytical solutions for the de-projected de Vaucouleurs and Sérsic laws using formal integration with Mathematica as well as for other related quantities like the mass or the potential, total potential energy and the central velocity dispersion.

2. Comparisons with existing numerical estimates show very few differences, however analytical expressions are always much more very convenient to deal with in many cases.

Acknowledgements. This work was supported by the BrazilFrance CNRS-CNPq cooperation. H.V.C acknowledges partial support from FAPESP (Project No. 2000/06695-2).

We warmly thank the referee, Dr. Mathews Colless, for very useful comments.

\section{Appendix A}

The projected luminosity profile is defined by:

$$
\begin{aligned}
L(R) & \equiv 2 \pi \int_{0}^{R} I\left(R^{\prime}\right) R^{\prime} \mathrm{d} R^{\prime} \\
& \equiv 2 \pi I_{0} R_{\mathrm{e}}^{2} \int_{0}^{x=R / R_{\mathrm{e}}} i\left(x^{\prime}\right) x^{\prime} \mathrm{d} x^{\prime} .
\end{aligned}
$$

In terms of the dimensionless quantities, $x \equiv R / R_{\mathrm{e}}$ and $l(x) \equiv L / I_{0} R_{\mathrm{e}}^{2}$, this gives:

$$
\begin{aligned}
l(x) & =\frac{2 m \pi e^{b}}{b^{2 m}} \gamma\left(2 m, b x^{\frac{1}{m}}\right) \\
& \equiv \frac{2 m \pi e^{b}}{b^{2 m}}\left[\Gamma(2 m)-\Gamma\left(2 m, b x^{\frac{1}{m}}\right)\right]
\end{aligned}
$$

where $\gamma(a, x)$ is the incomplete gamma function and $\Gamma$ its complement (cf. Gradshteyn \& Ryzhik 1980). For integer values of $m$ it can also be expressed as:

$$
\begin{aligned}
& l(x)=\frac{2 m \pi e^{b}}{b^{2 m}} \cdot\left[(2 m-1) !-\exp \left(-b x^{1 / m}\right)\right. \\
& \left.\times\left((2 m-1) !+\sum_{j=1}^{2 m-1} \frac{(2 m-1) !}{(2 m-j) !} b^{2 m-j} x^{(2 m-j) / m}\right)\right] .
\end{aligned}
$$

From this one may find $b(m)$ as the solutions of the equation $L\left(R_{\mathrm{e}}\right) \equiv L_{\text {tot }} / 2$, where $L_{\text {tot }}$ is the total luminosity integrated to infinity. We have (see also Ciotti 1991):

$\gamma(2 m, b) \equiv \Gamma(2 m, b)=\Gamma(2 m) / 2$.

This is solved instantaneously using the following Mathematica commands:

$\mathrm{mm}=\{1,2,3,4,5,6,7,8,9,10,11,12,13,14,15\}$

$\mathrm{b}\left[\mathrm{m}_{-}\right]:=$FindRoot $[$Gamma $[2 \mathrm{~m}, \mathrm{~b}]==\operatorname{Gamma}[2 \mathrm{~m}] / 2,\{\mathrm{~b}, 2 \mathrm{~m}-$

$1 / 3\}$, WorkingPrecision $\rightarrow 60$, AccuracyGoal $\rightarrow 30]$

blist $=\operatorname{Map}[b, \mathrm{~mm}] ; \mathrm{bb}=\mathrm{N}[\mathrm{b} /$. blist, 17]

The derived values of $b(m)$ are given in Table A.1, where we compare the Mathematica results with those from the asymptotic expansions by Ciotti \& Bertin (1999). For $m=$ 4 , we find of course $b(4)=7.66925$.

Table A.1. The values of $b(m)$.

\begin{tabular}{rccc}
\hline$m$ & $b(m)$ & $b_{\mathrm{CB} 99}$ & $b(m)-b_{\mathrm{CB} 99}$ \\
\hline 1 & 1.67834699001666 & 1.67838865492157 & $-4.16649049 \times 10^{-5}$ \\
2 & 3.67206074885089 & 3.67206544591768 & $-4.6970667 \times 10^{-6}$ \\
3 & 5.67016118871207 & 5.67016250849902 & $-1.3197869 \times 10^{-6}$ \\
4 & 7.6692494425008 & 7.66924998466950 & $-5.421687 \times 10^{-7}$ \\
5 & 9.66871461471413 & 9.66871488808778 & $-2.733736 \times 10^{-7}$ \\
6 & 11.6683631530448 & 11.6683633097115 & $-1.566667 \times 10^{-7}$ \\
7 & 13.6681145993449 & 13.6681146973462 & $-9.80013 \times 10^{-8}$ \\
8 & 15.6679295443172 & 15.6679296096535 & $-6.53363 \times 10^{-8}$ \\
9 & 17.6677864177885 & 17.6677864635090 & $-4.57206 \times 10^{-8}$ \\
10 & 19.6676724233057 & 19.6676724565414 & $-3.32357 \times 10^{-8}$ \\
11 & 21.6675794898319 & 21.6675795147457 & $-2.49138 \times 10^{-8}$ \\
12 & 23.6675022752263 & 23.6675022943807 & $-1.91544 \times 10^{-8}$ \\
13 & 25.6674371029624 & 25.6674371180047 & $-1.50423 \times 10^{-8}$ \\
14 & 27.6673813599995 & 27.6673813720274 & $-1.20280 \times 10^{-8}$ \\
15 & 29.6673331382212 & 29.6673331479896 & $-9.7684 \times 10^{-9}$ \\
\hline
\end{tabular}

The luminosity so writes:

- for $m=2$, with $b=b(2)$ :

$\frac{4 \pi}{b^{4}} e^{b}\left[6-e^{-b \sqrt{x}}\left(6+6 b \sqrt{x}+3 b^{2} x+b^{3} x^{\frac{3}{2}}\right)\right]$

- for $m=4$, with $b=b(4)$ :

$$
\begin{aligned}
& \frac{8 \pi}{b^{8}} e^{b}\left[5040-e^{-b x^{\frac{1}{4}}}\left(5040+5040 b x^{\frac{1}{4}}+2520 b^{2} \sqrt{x}\right.\right. \\
& \left.\left.\quad+840 b^{3} x^{\frac{3}{4}}+210 b^{4} x+42 b^{5} x^{\frac{5}{4}}+7 b^{6} x^{\frac{3}{2}}+b^{7} x^{\frac{7}{4}}\right)\right](\text { A. } 6)
\end{aligned}
$$

and so on ... 


\section{References}

Ciotti, L. 1991, A\&A, 249, 99

Ciotti, L., \& Bertin, G. 1999, A\&A, 353, 447

de Vaucouleurs, G. 1948, Ann. Astroph., 11, 247

Fuchs, B., \& Materne, J. 1982, A\&A, 113, 85

Gradshteyn, I. S., \& Ryzhik, I. M. 1980, Table of Integrals, Series and Products (Academic Press)

Graham, A., \& Colless, M. 1997, MNRAS, 287, 221
Marquez, I., Lima Neto, G. B., Capelato, H. V., et al. 2001, A\&A, 379, 767

Mellier, Y., \& Mathez, G. 1987, A\&A, 175, 1

Poveda, A., Iturriaga, R., \& Orozco, I. 1960, Bol. Obs. Tonantzinla N. 20, 3

Sérsic, J.-L. 1968, Atlas de Galaxias Australes, Observatorio Astronomico de Cordoba

Young, P. J. 1976, AJ, 81, 807

Wolfram, S. 1991, The Mathematica Handbook (AddisonWesley Publishing Company, Inc.) 